\title{
Relativistic Mean-Field Models and Nuclear Matter Constraints
}

\author{
M. Dutra*, O. Lourenço*, B. V. Carlson*, A. Delfino ${ }^{\dagger}$, D. P. Menezes**, \\ S. S. Avancini** J. R. Stone ${ }^{\ddagger}$, , C. Providência ${ }^{\text {qI }}$ and S. Typel ${ }^{\|}$ \\ *Departamento de Física, Instituto Tecnológico de Aeronáutica-CTA, 12228-900, São José dos \\ Campos, SP, Brazil \\ ${ }^{\dagger}$ Instituto de Física, Universidade Federal Fluminense, 24210-150, Boa Viagem, Niterói, RJ, Brazil \\ ** Departamento de Física, CFM, Universidade Federal de Santa Catarina, CP. 476, CEP \\ 88.040-900, Florianópolis, SC, Brazil \\ $\ddagger$ Oxford Physics, University of Oxford, OX1 3PU Oxford, United Kingdom \\ ${ }^{\S}$ Department of Physics and Astronomy, University of Tennessee, Knoxville, Tennessee 37996, USA \\ "Centro de Física Computacional, Department of Physics, University of Coimbra, P-3004-516 \\ Coimbra, Portugal \\ " GSI Helmholtzzentrum für Schwerionenforschung GmbH, Theorie, Planckstrasse 1,D-64291 \\ Darmstadt, Germany
}

\begin{abstract}
This work presents a preliminary study of 147 relativistic mean-field (RMF) hadronic models used in the literature, regarding their behavior in the nuclear matter regime. We analyze here different kinds of such models, namely: (i) linear models, (ii) nonlinear $\sigma^{3}+\sigma^{4}$ models, (iii) $\sigma^{3}+\sigma^{4}+\omega^{4}$ models, (iv) models containing mixing terms in the fields $\sigma$ and $\omega$, (v) density dependent models, and (vi) point-coupling ones. In the finite range models, the attractive (repulsive) interaction is described in the Lagrangian density by the $\sigma(\omega)$ field. The isospin dependence of the interaction is modeled by the $\rho$ meson field. We submit these sets of RMF models to eleven macroscopic (experimental and empirical) constraints, used in a recent study in which 240 Skyrme parametrizations were analyzed. Such constraints cover a wide range of properties related to symmetric nuclear matter (SNM), pure neutron matter (PNM), and both SNM and PNM.
\end{abstract}

Keywords: relativistic models, asymmetric nuclear matter, constraints

PACS: 21.30.Fe, 21.65.Cd, 21.65.Ef

Quantum Hadrodynamics (QHD) is an approach based on quantum field theory much used in the description of hadronic environments, such as nuclear and neutron matter. It is based on local Lagrangian densities whose free parameters are adjusted in order to reproduce basic nuclear matter bulk properties at zero temperature. In general, nuclear matter is well described by different versions of the relativistic mean-field (RMF) models constructed via the QHD approach.

In order to select a set of RMF models which better describe nuclear matter properties, as well as the physics of pure neutron matter, we submit 147 relativistic parameterizations to 11 constraints, out of which 4 are related to symmetric nuclear matter, 2 to pure neutron matter, and 5 related to the symmetry energy that involve both symmetric and pure neutron matter. All these constraints were taken at the saturation point $\left(\rho_{0}\right)$, except the constraints related with the band region. For more details about the constraints and the criteria used for approval see Ref. [1].

The relativistic hadronic models $[2,3,4]$ used here are described by the following Lagrangian densities: 
(a) Nonlinear finite range (containing 123 models):

$$
\mathscr{L}_{\mathrm{NL}}=\mathscr{L}_{\mathrm{nm}}+\mathscr{L}_{\sigma}+\mathscr{L}_{\omega}+\mathscr{L}_{\rho}+\mathscr{L}_{\sigma \omega \rho}
$$

where

$$
\begin{aligned}
\mathscr{L}_{\mathrm{nm}} & =\bar{\psi}\left(i \gamma^{\mu} \partial_{\mu}-M\right) \psi+g_{\sigma} \sigma \bar{\psi} \psi-g_{\omega} \bar{\psi} \gamma^{\mu} \omega_{\mu} \psi-\frac{g_{\rho}}{2} \bar{\psi} \gamma^{\mu} \vec{\rho}_{\mu} \cdot \vec{\tau} \psi, \\
\mathscr{L}_{\sigma} & =\frac{1}{2}\left(\partial^{\mu} \sigma \partial_{\mu} \sigma-m_{\sigma}^{2} \sigma^{2}\right)-\frac{A}{3} \sigma^{3}-\frac{B}{4} \sigma^{4}, \\
\mathscr{L}_{\omega} & =-\frac{1}{4} F^{\mu v} F_{\mu \nu}+\frac{1}{2} m_{\omega}^{2} \omega_{\mu} \omega^{\mu}+\frac{c}{4}\left(g_{\omega}^{2} \omega_{\mu} \omega^{\mu}\right)^{2}, \\
\mathscr{L}_{\rho} & =-\frac{1}{4} \vec{B}^{\mu v} \cdot \vec{B}_{\mu v}+\frac{1}{2} m_{\rho}^{2} \vec{\rho}_{\mu} \cdot \vec{\rho}^{\mu} \text { and } \\
\mathscr{L}_{\sigma \omega \rho} & =g_{\sigma} g_{\omega}^{2} \sigma \omega_{\mu} \omega^{\mu}\left(\alpha_{1}+\frac{1}{2} \alpha_{1}^{\prime} g_{\sigma} \sigma\right)+g_{\sigma} g_{\rho}^{2} \sigma \vec{\rho}_{\mu} \cdot \vec{\rho}^{\mu}\left(\alpha_{2}+\frac{1}{2} \alpha_{2}{ }^{\prime} g_{\sigma} \sigma\right) \\
& +\frac{1}{2} \alpha_{3}{ }^{\prime} g_{\omega}^{2} g_{\rho}^{2} \omega_{\mu} \omega^{\mu} \vec{\rho}_{\mu} \cdot \vec{\rho}^{\mu} .
\end{aligned}
$$

(b) Density dependent models (containing 6 models):

$$
\begin{aligned}
\mathscr{L}_{\mathrm{DD}} & =\bar{\psi}\left(i \gamma^{\mu} \partial_{\mu}-M\right) \psi+\Gamma_{\sigma}(\rho) \sigma \bar{\psi} \psi-\Gamma_{\omega}(\rho) \bar{\psi} \gamma^{\mu} \omega_{\mu} \psi-\frac{\Gamma_{\rho}(\rho)}{2} \bar{\psi} \gamma^{\mu} \vec{\rho}_{\mu} \cdot \vec{\tau} \psi \\
& +\frac{1}{2}\left(\partial^{\mu} \sigma \partial_{\mu} \sigma-m_{\sigma}^{2} \sigma^{2}\right)-\frac{1}{4} F^{\mu v} F_{\mu \nu}+\frac{1}{2} m_{\omega}^{2} \omega_{\mu} \omega^{\mu}-\frac{1}{4} \vec{B}^{\mu \nu} \cdot \vec{B}_{\mu \nu}+\frac{1}{2} m_{\rho}^{2} \vec{\rho}_{\mu} \cdot \vec{\rho}^{\mu}
\end{aligned}
$$

where $\Gamma_{i}(\rho)=\Gamma_{i}\left(\rho_{0}\right) f_{i}(x)$, with $f_{i}(x)=a_{i} \frac{1+b_{i}\left(x+d_{i}\right)^{2}}{1+c_{i}\left(x+d_{i}\right)^{2}}$ for $i=\sigma, \omega$, and $\Gamma_{\rho}(\rho)=\Gamma_{\rho}\left(\rho_{0}\right) e^{-a(x-1)}$ with $x=\rho / \rho_{0}$.

In both Lagrangian densities, $M, m_{i}$, with $i=\sigma, \omega$ and $\rho$ are the nucleon and the mesons masses, respectively. The tensor fields are given by $F_{\mu \nu}=\partial_{\nu} \omega_{\mu}-\partial_{\mu} \omega_{\nu}$ and $\vec{B}_{\mu \nu}=\partial_{\nu} \vec{\rho}_{\mu}-\partial_{\mu} \vec{\rho}_{\nu} \cdot g_{i}(i=\sigma, \omega$, and $\rho), A$, and $B$ are the coupling constants.

(c) Point-coupling models (containing 18 models):

$$
\begin{aligned}
\mathscr{L}_{\mathrm{PC}} & =\bar{\psi}\left(i \gamma^{\mu} \partial_{\mu}-M\right) \psi-\frac{\alpha_{S}}{2}(\bar{\psi} \psi)^{2}-\frac{\beta_{S}}{3}(\bar{\psi} \psi)^{3}-\frac{\gamma_{S}}{4}(\bar{\psi} \psi)^{4} \\
& -\frac{\alpha_{V}}{2}\left(\bar{\psi} \gamma^{\mu} \psi\right)^{2}-\frac{\gamma_{V}}{4}\left(\bar{\psi} \gamma^{\mu} \psi\right)^{4}-\frac{\alpha_{T V}}{2}\left(\bar{\psi} \gamma^{\mu} \vec{\tau} \psi\right)^{2}-\frac{\gamma_{T V}}{4}\left(\bar{\psi} \gamma^{\mu} \vec{\tau} \psi\right)^{4} \\
& -\frac{\alpha_{T S}}{2}(\bar{\psi} \vec{\tau} \psi)^{2}+\left[\eta_{1}+\eta_{2}(\bar{\psi} \psi)\right](\bar{\psi} \psi)\left(\bar{\psi} \gamma^{\mu} \psi\right)^{2}-\eta_{3}(\bar{\psi} \psi)\left(\bar{\psi} \gamma^{\mu} \vec{\tau} \psi\right)^{2},
\end{aligned}
$$

where $\alpha_{S}, \beta_{S}, \gamma_{S}, \alpha_{V}, \gamma_{V}, \alpha_{T V}, \gamma_{T V}, \alpha_{T S}, \eta_{1}, \eta_{2}, \eta_{3}$ are the coupling constants. The subscripts identify the coupling: $S$ stands for scalar, $V$ for vector, and $T$ for isovector.

The equations of state for these groups, e.g., the energy density and pressure, are calculated from the energy-momentum tensor (in the mean-field approximation): $\mathscr{E}=\left\langle T_{00}\right\rangle$, and $P=\frac{1}{3}\left\langle T_{i i}\right\rangle$ respectively. Other quantities, such as the symmetry energy, can be obtained from the energy density or the pressure.

These equations are calculated for each density and proton fraction, $\rho$ and $y=\frac{Z}{A}$ respectively, from the simultaneous solution of the field equations (obtained from the Euler-Lagrange ones). 
The results obtained were the following: out of 147 models only 9 of them satisfies all the constraints, excluding the constraint related to $K_{\tau, \mathrm{v}}$ (isospin dependence of the incompressibility) [1]. These are: BSR15, BSR16, BSR17 (5\% of tolerance in the PNM2 constraint - see [1]), BSR18 (5\% of tolerance in the PNM2 constraint - see [1]) [2], DD-F, [5] FSUGold, [6] FSUGold4, [7] FSUGZ06, [8] and TW99 [3]. Their nuclear matter properties are given in Table 1. It is important to mention that among these RMF models, 7 are nonlinear with mixing terms in the $\sigma$ and $\omega$ fields, identified with a $(*)$ and 2 are density dependent, marked with a $(* *)$.

TABLE 1. Properties of nuclear matter at saturation density as calculated using the RMF models consistent with the macroscopic constraints. All entries are in $\mathrm{MeV}$.

\begin{tabular}{lcccccccc}
\hline Model & $\rho_{\mathbf{0}}$ & $\mathbf{E}_{\mathbf{0}}$ & $\mathbf{K}_{\mathbf{0}}$ & $\mathbf{m}^{*}$ & $\mathbf{K}^{\prime}$ & $\mathbf{J}$ & $\mathbf{L}$ & $\mathbf{K}_{\tau, \mathrm{v}}$ \\
\hline BSR15* $^{*}$ & 0.146 & -16.03 & 226.82 & 0.61 & 512.29 & 30.97 & 61.79 & -252.54 \\
BSR16* $^{*}$ & 0.146 & -16.05 & 224.98 & 0.61 & 503.17 & 31.24 & 62.33 & -258.75 \\
BSR17* $^{*}$ & 0.146 & -16.05 & 221.67 & 0.61 & 489.45 & 31.98 & 67.44 & -287.31 \\
BSR18* $^{*}$ & 0.146 & -16.05 & 221.13 & 0.61 & 485.73 & 32.74 & 72.65 & -318.55 \\
DD-F** $^{*}$ & 0.147 & -16.04 & 223.32 & 0.56 & 758.73 & 31.63 & 56.00 & -285.54 \\
FSUGold $^{*}$ & 0.148 & -16.28 & 229.54 & 0.61 & 523.93 & 32.56 & 60.44 & -276.07 \\
FSUGold4 $^{*}$ & 0.147 & -16.40 & 229.56 & 0.61 & 538.33 & 31.40 & 51.74 & -205.59 \\
FSUGZ06 $^{*}$ & 0.146 & -16.05 & 225.06 & 0.61 & 503.17 & 31.18 & 62.42 & -259.47 \\
TW99** & 0.153 & -16.25 & 240.27 & 0.55 & 539.79 & 32.77 & 55.31 & -332.32 \\
\hline
\end{tabular}

As in the previous work [1], we see that very few models among the initial 147 satisfy all the constraints used. We note (see Table 1) that the density-dependent models have values of nucleon effective mass different from the others. We also observe that if we include in the set of constraints the ones related to the isospin dependence of the incompressibility, then none of the models are consistent. Because of this, the behavior of these models with respect to $K_{\tau, \mathrm{v}}$ should be better investigated.

As a final remark, we highlight the importance of the present study in the identification of the relativistic parametrizations which better describe the physics of both nuclear and pure neutron matter.

\section{ACKNOWLEDGMENTS}

M.D. and B.V.C. acknowledge support from FAPESP. S.S.A., B.V.C., A.D. and D.P.M. acknowledge support from the CNPq.

\section{REFERENCES}

1. M. Dutra, O. Lourenço, J. S. Sá Martins, A. Delfino, J. R. Stone, P. D. Stevenson, Phy. Rev. C, 85, 035201 (2012).

2. S. K. Dhiman, R. Kumar, and B. K. Agrawal, Phys. Rev. C 76, 045801 (2007).

3. S. Typel, and H. H. Wolter, Nucl. Phys. A656, 331 (1999).

4. T. Bürvenich, et al., Phys. Rev. C 65, 044308 (2002).

5. T. Klähn, et al., Phys. Rev. C 74, 035802 (2006).

6. B. G. T. Rutel, and J. Piekarewicz, Phys. Rev. Lett. 95, 122501 (2005).

7. J. Piekarewicz, and S. P. Weppner, Nucl. Phys. A778, 10 (2006).

8. R. Kumar, B. K. Agrawal, and S. K. Dhiman, Phys. Rev. C 74, 034323 (2006). 